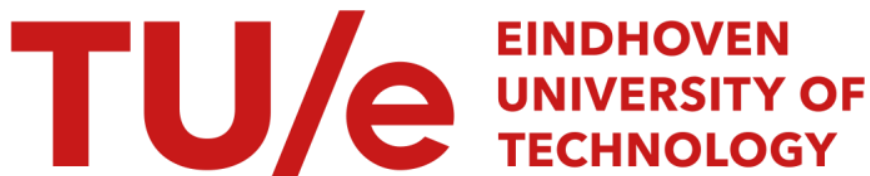

\section{Shear Thickening (Dilatancy) in Concentrated Dispersions}

\section{Citation for published version (APA):}

Boersma, W. H., Laven, J., \& Stein, H. N. (1990). Shear Thickening (Dilatancy) in Concentrated Dispersions. AlChE Journal, 36(3), 321-332. https://doi.org/10.1002/aic.690360302

DOI:

10.1002/aic.690360302

Document status and date:

Published: 01/01/1990

\section{Document Version:}

Publisher's PDF, also known as Version of Record (includes final page, issue and volume numbers)

\section{Please check the document version of this publication:}

- A submitted manuscript is the version of the article upon submission and before peer-review. There can be important differences between the submitted version and the official published version of record. People interested in the research are advised to contact the author for the final version of the publication, or visit the $\mathrm{DOI}$ to the publisher's website.

- The final author version and the galley proof are versions of the publication after peer review.

- The final published version features the final layout of the paper including the volume, issue and page numbers.

Link to publication

\section{General rights}

Copyright and moral rights for the publications made accessible in the public portal are retained by the authors and/or other copyright owners and it is a condition of accessing publications that users recognise and abide by the legal requirements associated with these rights.

- Users may download and print one copy of any publication from the public portal for the purpose of private study or research.

- You may not further distribute the material or use it for any profit-making activity or commercial gain

- You may freely distribute the URL identifying the publication in the public portal.

If the publication is distributed under the terms of Article 25fa of the Dutch Copyright Act, indicated by the "Taverne" license above, please follow below link for the End User Agreement:

www.tue.nl/taverne

Take down policy

If you believe that this document breaches copyright please contact us at:

openaccess@tue.nl

providing details and we will investigate your claim. 


\section{Shear Thickening (Dilatancy) in Concentrated Dispersions}

\begin{abstract}
A new criterion is introduced for the onset of shear thickening in a concentrated dispersion. The criterion follows from the assumption that shear thickening occurs when the shear forces overrule the interparticle forces. A force balance at small interparticle distances is used to predict the dependence of this critical shear rate on the volume fraction. It is deduced that the critical shear rate is proportional to the interparticle distance, has a linear dependence on the magnitude of the stabilizing force, an inverse linear dependence on the dispersion medium viscosity, and an inverse linear dependence on the particle radius. The model is confirmed experimentally with viscosity measurements on various electrostatically stabilized dispersions. The validity of the model is also checked with data obtained from the literature. Experiments indicate that polydisperse dispersions exhibit pronounced shear thickening but with a less dramatic increase in viscosity than monodisperse dispersions.
\end{abstract}

\author{
Willem H. Boersma \\ Jozua Laven \\ Hans N. Stein \\ Department of Colloid Chemistry \\ Technische Universiteit Eindhoven \\ 5600 MB Eindhoven, The Netherlands
}

\section{Introduction}

Colloidal dispersions are used in various industries, of which the food, paint, and ceramic industries are only three examples. For processing the dispersions, knowledge of the flow behavior is one of the main aspects that must be understood. This applies especially to concentrated dispersions.

The rheology of concentrated dispersions has been the subject of continuing research since the beginning of this century. In the last twenty years, interest in the subject increased greatly and more attention was paid to the description and understanding of shear thickening in concentrated dispersions. It was discovered, for example (Hoffman, 1972), that concentrated plastisols of monodisperse polyvinylchloride particles show discontinuous viscosity behavior. In this case the flow curve shows a sudden jump in viscosity at a certain critical shear rate. With other dispersions such a jump, or a more gradual increase in viscosity over a range of shear rates, occurs (Alince and Lepoutre, 1983; Hoffman, 1982; Metzner and Whitlock, 1958; Pätzold, 1980; Strivens, 1976; Wagstaff and Chaffey, 1977; Willey and Macosko, 1978). Different explanations have been given for the phenomenon of shear thickening in monodisperse dispersions but it is now almost generally accepted that an order-disorder

Correspondence concerning this paper should be addressed to W. H. Boersma. transition is responsible for the rise in viscosity. This can be concluded from light scattering experiments as well as from computer simulations. With polydisperse, shear thickening dispersions the situation is less clear.

Hoffman (1972) showed with light scattering that when shearing well-stabilized monodisperse polyvinylchloride dispersions in dioctylphtalate, a transition occurred from a two-dimensional ordered state of hexagonally packed layers to a disordered state. This transition appeared at the same shear rate at which the sudden jump in viscosity took place. More recently it was also shown with light diffraction techniques (Tomita and van de Ven, 1984) that, under shear, monodisperse latices can flow in an ordered two-dimensional lattice structure up to relatively high shear rates. In the systems investigated only the distance between the layers of particles increased slightly with increasing shear rate. The authors were able to give a qualitative explanation of their observations by considering the hydrodynamic and colloidal forces acting on a particle. They did not observe, however, an order-disorder transition with their dispersions in the shear rate range measured.

Most of the computer simulations that show an order-disorder transition have involved atomic fluids (Barnes et al., 1987; Denisov et al., 1985; Heyes, 1986; Loose and Hess, 1989) but recently a method was introduced by Durlofsky et al. (1987) to perform simulations on concentrated colloidal dispersions. In 
these simulations hydrodynamic interactions and interparticle forces are taken into account. From the simulations on stabilized dispersions it can be seen that the viscosity increases with increasing shear rate (Brady and Bossis, 1985). This increase is, however, by far not as severe as is seen in experiments. The reason for this difference may be that the simulated dispersions were not concentrated enough, that the simulations were twodimensional, or that the level of the interparticle forces was different from practical situations. However, in the simulations it appeared that the state of the dispersions changed from a certain degree of order to clusters of particles that were formed because of the shear forces. These clusters were responsible for the increase in viscosity. Woodcock (1984) showed by molecular dynamics calculations that shear thickening occurs in classical dense fluids. He derived a scaling of the flow curve with diameter to the 2.5 power.

Recently, Barnes (1989) published a review article on shear thickening in which he concluded that shear thickening takes place in all dispersions. But only with some well-chosen dispersions does it occur at a shear rate measurable in the current commercial rheometers. According to Barnes the parameters that control shear thickening behavior are: particle size and particle size distribution, particle volume fraction, particle shape, particle-particle interaction, continuous phase viscosity, and the type, rate, and time of deformation. All of these parameters have been studied to some extent. Using some well-described data from recent experiments, Barnes showed that a consistent description of the phenomenon is now possible. However, very few calculations have been performed to predict flow curves of shear thickening dispersions and the shear rate at which shear thickening starts.

Hoffman (1974) presented a torque and kinetic energy balance in which he included shear forces, van der Waals-London attraction forces, and electrical double layer repulsion. On the basis of optical diffraction patterns, he started from the supposition of hexagonally packed layers of particles sliding over each other. He considered a doublet in a layer and calculated at which shear rate the above-mentioned forces made the doublet rotate out of its layer. At that shear rate the layered structure is unstable and the viscosity increases strongly. After elaborate calculations he was able to predict the onset of instability. Because of the limitations of his approximations, however, he did not make a rigorous test of the theory. But from experiments he concluded that especially the shear stress and the electrical double layer repulsion are very important in the process of shear thickening.

Berezov et al. (1982) concluded that changes in the balance between hydrodynamic and interparticle forces were responsible for the complex thixotropic-dilatant properties of the plastisols they investigated. But they did not perform a rigorous test of their theory either and their criterion is rather complicated to use as well.

In this article we present an easy to handle criterion that can, with reasonable accuracy, predict the shear rate at which shear thickening starts for different dispersions and which gives insight into the physical behavior of the system. It contains aspects from Hoffman's analysis and from the force balance of Berezov but is mathematically much more simple. The dependences on particle size, continuous phase viscosity, and particleparticle interaction are taken into account. We further indicate how the critical shear rate for shear thickening depends on the volume fraction of the solid phase. Experimental results confirm the model. Finally, it is shown that polydisperse dispersions can also exhibit pronounced shear thickening.

\section{Theory}

A general criterion is developed that can predict the shear rate at which shear thickening occurs as a function of volume fraction. This criterion is applicable to sterically or electrostatically stabilized dispersions. The critical shear rate is indicated by $\dot{\gamma}_{s}$.

We assume that when shearing a concentrated stabilized monodisperse dispersion at relatively low shear rates, repulsive interparticle forces (electrostatic or steric) keep the particles in a layered structure. At a certain higher shear rate, however, the shear forces that push the particles together become larger in magnitude than the interparticle forces. As a result of this, the particles are moved from their equilibrium position. This leads to a transition from a layered to a disordered structure, causing an increase of the dispersion viscosity.

In a more thorough treatment of the phenomenon, van der Waals attraction and Brownian motion should be taken into account. At a distance determined by a fixed volume fraction the energies of these two mechanisms are independent of particle size. On the other hand the energy of electrostatic repulsion scales more or less with particle size (see below). Thus neglecting van der Waals attraction and Brownian motion restricts the applicability of our theory to larger particles.

Within the limits indicated, the critical shear rate, $\dot{\gamma}_{s}$, at which shear thickening starts is the shear rate at which the magnitudes of the interparticle and the shear forces balance. From experiments it is seen that the magnitude of $\dot{\gamma}_{s}$ decreases with increasing volume fraction. We will develop an equation for $\dot{\gamma}_{s}$ at high volume fractions (and thus small interparticle distances $h$ ) by using the formulas for the electrostatic interaction between two particles and for the hydrodynamic interaction between two particles approaching each other along their line of centers.

For the electrostatic repulsion this formula is (Verwey and Overbeek, 1948):

$$
F_{\text {rep }}=2 \pi \epsilon_{0} \epsilon_{r} \psi_{0}^{2} \frac{\kappa a \exp (-\kappa h)}{1 \pm \exp (-\kappa h)}
$$

Here $\epsilon_{r}$ is the relative dielectric constant of the medium and $\epsilon_{0}$ is the permittivity of vacuum; $\psi_{0}$ is the surface potential, which can be approximated by the $\zeta$-potential of the particles in the medium; $\eta_{0}$ is the medium viscosity; $a$ is the particle radius; and $\kappa$ is the reciprocal Debye double layer thickness. For a symmetrical electrolyte the value of $\alpha$ is given by (Kruyt, 1952):

$$
\kappa=\sqrt{\frac{2 C N_{A V z^{2} e_{0}^{2}}}{\epsilon_{0} \epsilon_{r} k T}}
$$

In this formula $C$ is the concentration of electrolyte, $N_{A V}$ is Avogadro's constant, $z$ is the valence of the ions, $e_{0}$ is the elementary unit of charge, $k$ is Boltzmann's constant, and $T$ is the absolute temperature.

In Eq. $1 h$ is the distance between the two particles. The + and - in Eq. 1 correspond to constant surface potential $\psi_{0}$ and constant surface charge density of the spheres. If we assume 
constant surface potential for small $h$, Eq. 1 reduces to:

$$
F_{\text {rep }}=2 \pi \epsilon_{0} \epsilon_{r} \psi_{0}^{2} \kappa a / 2
$$

The choice for constant surface potential is based on the fact that in a concentrated dispersion the particles are continuously at relative small distances to neighbors. These distances vary with time. Given enough time, the particles will adjust their surface potential to this situation until the equilibrium value is reached. Frens and Overbeek (1972) showed (on the assumption that the transport of ions from the absorbed to the dissolved state is determined by diffusion in the liquid) that in water the diffusion time estimated for adjusting the surface potential to a change in particle distance is on the order of one second. In glycerol/water, with a viscosity 140 times that of water, this would be on the order of a few minutes. But since we are shearing, the time for the adaptation of the surface potential will be significantly shorter due to convection.

For the hydrodynamic interaction between two spheres approaching each other along their line of centers, Frankel and Acrivos (1967) showed that the formulation of Brenner (1961) for a sphere approaching a free surface could be used. For small values of $h$ this gives for two spheres of equal size:

$$
F_{\text {hydrodyn }}=6 \pi \eta_{0} a^{2} \dot{\gamma} a / h
$$

If these two forces are equated, one obtains for $\dot{\gamma}_{s}$ :

$$
\dot{\gamma}_{s}=\frac{2 \pi \epsilon_{0} \epsilon_{r} \psi_{0}^{2}}{6 \pi \eta_{0} a^{2}} \frac{\kappa h}{2}
$$

Equation 5 indicates that in this model $\dot{\gamma}_{s}$ is proportional to the distance between the particles.

The simplification in the limit of small $h$ in Eqs. 3 and 4 in principle restricts the validity of Eq. 5 to $h / a<0.05( \pm 25 \%$ error), which, as we shall see, equals $\phi \approx 0.56$. However, the expression for the relative viscosity derived by Frankel and Acrivos on the basis of this simplification of Eq. 4 is seen to compare well with experimental results down to volume fractions of about 0.4 , which suggests that the limit for small $h$ is usable in a wider range. It must be noted that the method of Frankel and Acrivos has been criticized (Marrucci and Denn, 1985). One of the criticisms is that the use of two-particle interactions in a concentrated dispersion is questionable. For electrostatic interactions this is probably a reasonable assumption but for the hydrodynamic interactions it is known to be a very crude one. It is, however, the most usable approximation and we think that for a simplified analysis like ours it is reasonable.

Since we are also interested in the relationship between $\dot{\gamma}_{s}$ and $\phi$ we must deduce the relationship between the interparticle distance $h$ and $\phi$. This can be done in various ways. In Appendix A the results of different analyses are presented. We choose a method based on the assumption that the smallest distance between two particles in a hexagonally packed layer is equal to the smallest distance between particles in two adjacent layers. This distance is a function of volume fraction and is given by:

$$
h / a=\left(\frac{8 \pi}{3 \sqrt{3} \phi}\right)^{1 / 3}-2
$$

Considering Eq. 5, we see that $\dot{\gamma}_{s}$ depends on the volume fraction according to:

$$
\dot{\gamma}_{s}\left(\phi_{1}\right)=h\left(\phi_{1}\right) / h\left(\phi_{2}\right) \cdot \dot{\gamma}_{s}\left(\phi_{2}\right)
$$

where $h\left(\phi_{1}\right)$ and $h\left(\phi_{2}\right)$ are respectively related to $\phi_{1}$ and $\phi_{2}$ through Eq. 6. Equations 5-7 are referred to henceforth as the refined model.

It is sometimes convenient to have an even more simple estimate of the shear rate at which shear thickening will appear, for example, a dimensionless number from which it can be seen very easily at what shear rate range shear thickening is to be expected for a given dispersion. This is then independent of the volume fraction. For this it is sufficient to use the characteristic magnitudes of the interparticle and shear forces (Russel, 1980; Bossis and Brady, 1984). Then the following dimensionless number is found:

$$
N_{d}=\frac{6 \pi \eta_{0} a^{2} \dot{\gamma}}{2 \pi \epsilon_{0} \epsilon_{r} \psi_{0}^{2}}
$$

Shear thickening will occur at values of $N_{d}$ larger than approximately one. This dimensionless number is equal to the dimensionless shear rate defined by Bossis and Brady (1984) for their computer simulations. In Appendix B a short discussion of this simple model is presented together with a choice of a volume fraction at which it is best used. We also show there the close resemblance between the simple model and an approach based on the assumption of constant surface charge. In Appendix C the simple model is presented for sterically stabilized dispersions, together with a discussion of the problem of the cooperation of different types of stabilization on one particle.

The following sections describe model testing with experiments carried out with electrostatically stabilized concentrated dispersions.

\section{Experimental Method}

Rheological measurements were performed on dispersions of:

- Polyvinylchloride (PVC) in dioctylphtalate (DOP)

- Polystyrene (PS) in water

- Polystyrene in a glycerol/water $(86.1 / 13.9 \mathrm{w} / \mathrm{w})$ mixture

- Silica $\left(\mathrm{SiO}_{2}\right)$ in glycerol/water $(86.1 / 13.9 \mathrm{w} / \mathrm{w})$

- Glass particles in glycerol/water $(86.1 / 13.9 \mathrm{w} / \mathrm{w})$

Measurements were carried out with a Contraves Rheomat 115 shear rate controlled rheometer, supplied with a personal computer, using various concentric cyclinder systems in order to cover a wide range of shear stresses. During all measurements the rheometer was kept at $20.0 \pm 0.1^{\circ} \mathrm{C}$, except for the measurements of PVC in DOP $\left(25.0 \pm 0.1^{\circ} \mathrm{C}\right)$ and the series of measurements investigating temperature dependence. Measurements were made as quickly as possible. This means that a value for the torque at a constant shear rate was taken as soon as the torque reached its maximum value, in order to minimize thixotropic effects.

After the measuring system was filled, with care being taken not to include air in the system, the system was fitted into the rheometer. There it was first kept at rest for $30 \mathrm{~min}$ in order to equilibrate thermally and to restore possible structures that might have been destroyed during the filling of the system. The 
system was closed by a Teflon cover in order to prevent evaporation of the dispersion medium. Checks for wall slip were made by using the Couette two-measurement method (Yoshimura and Prud'homme, 1988) but no significant wall slip was measured. Checks were made at the highest volume fractions and up to the onset of shear thickening.

Dispersion samples were prepared in $100 \mathrm{~mL}$ wide-neck sample bottles by mixing the constituents by hand during addition of the particles until the correct volume fraction was reached. Then the samples were stirred mechanically with a two-blade propeller stirrer (blade dia. $3 \mathrm{~cm}$ ) at 2,000 rpm. Stirring was continued until, after careful dilution of the sample with dispersion medium, no aggregates could be seen under the microscope. The only exception to this were the polystyrene in water samples, which were prepared by evaporation of the liquid in a vacuum oven until approximately the desired volume fraction was reached. After a measurement the exact volume fraction was determined by drying a sample of known weight at $50^{\circ} \mathrm{C}$ in a vacuum oven and calculating the volume fraction from the weight fraction. These dispersions were also checked for aggregates under the microscope but none could be observed. For the dispersion of polystyrene in the glycerol/water mixture dried polystyrene was used and the normal procedure was followed. After preparation of the dispersions all samples were left for $2 \mathrm{~h}$ to equilibrate before measurement.

Particle sizes were determined with a Coulter Counter ZM 256, $\zeta$-potentials were measured with a Rank Brothers MK II, all densities were measured with pycnometers, and conductivities were measured with a Philips PW9505 conductivity tester. The concentration of electrolyte in the liquid was estimated by comparison of the conductivity of the supernatant of a dispersion with conductivities of solutions of $\mathrm{KCl}$ in the standard glycerol/water mixture with known concentrations. Only the measurements on polystyrene in water were compared to measurements on solutions of sodium dodecylsulfate in water.

Glass particles were obtained from Potters Ballotini (soda lime glass). They were washed twice with concentrated nitric acid and then washed twelve times with double-distilled water until the $\mathrm{pH}$ was constant $( \pm 9.5)$. Washing was performed by centrifugation, decanting the liquid, and resuspending in new liquid. After washing the particles were dried in a vacuum oven at $150^{\circ} \mathrm{C}$. The density of the particles was $2,550 \mathrm{~kg} / \mathrm{m}^{3}$. The particle size distribution, shown in Figure la, was rather wide. Electron microscope photographs revealed that some particles (about 5\%) were aspherical, although the majority were spherical. The number-average diameter was $2.4 \mu \mathrm{m}$ and the ratio of the particle weight average to the number-average diameter was 2.08 .

Polyvinylchloride (PVC) particles (Vinnol P70F) were obtained from Wacker Chemie and used as supplied. From Figure lb it can be seen that the size distribution was polydisperse. From electron micrographs it could be seen that the particles were spherical. The number-average diameter was $1.4 \mu \mathrm{m}$ and the density $1,390 \mathrm{~kg} / \mathrm{m}^{3}$. The ratio of the weight average to number-average diameter is 1.41 .

Silica $\left(\mathrm{SiO}_{2}\right)$ particles were prepared by the method of Tan et al. (1987) at $-20^{\circ} \mathrm{C}$ in a $10 \mathrm{~L}$ vessel stirred with a magnetic stirrer. $N$-propanol (Fluka AG) was used as solvent. Tetramethylorthosilicate ( $98 \%$, Janssen Chimica) was distilled before use. The mixture was kept at $-20^{\circ} \mathrm{C}$ for $16 \mathrm{~h}$ and the reaction yielded approximately $75 \mathrm{~g}$ of silica.
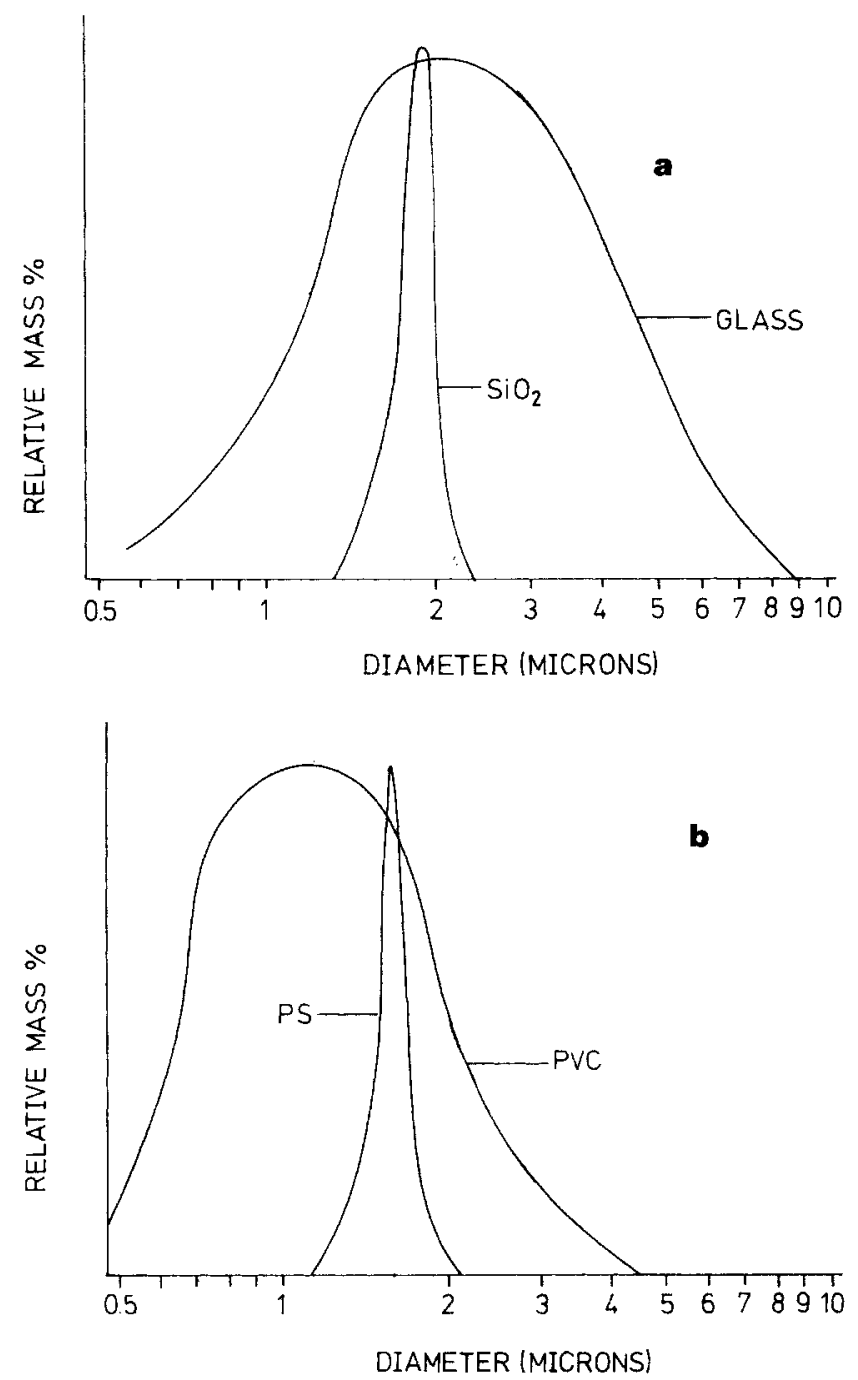

Figure 1. Particle size distributions.

a. Glass and $\mathrm{SiO}_{2}$

b. PS and PVC

The silica particles were first washed five times with doubledistilled water and then washed and dried in the same way as the glass particles. As can be seen from Figure 1a, they were fairly monodisperse. In electron micrographs it could be seen that a few particles were not completely spherical, but were slightly oval or showed small surface irregularities. The great majority, however, were spherical. The number-average diameter was $1.91 \mu \mathrm{m}$ and the ratio of the weight average to the number-average diameter was 1.06 . The density was $2,026 \mathrm{~kg} / \mathrm{m}^{3}$. However, from Ubbelohde viscosity measurements on dispersions of the silica particles in water at low volume fractions, using the Einstein relation for the relative viscosity, a hydrodynamic density of $1,457 \mathrm{~kg} / \mathrm{m}^{3}$ was obtained. This suggests that the particles are porous and that water can fill the pores. In mercury porosimetry measurements no pores could be observed with a pore diameter above $10 \mathrm{~nm}$. In electron micrographs the particle surfaces appeared to be smooth. We conclude therefore that there are very small pores in the particles which fill with water and that in determining the volume fraction we must use the hydrodynamic density. 
Polystyrene (PS) particles were prepared according to the method of Almog et al. (1982). We used $300 \mathrm{~mL}$ styrene (Merck, zur Synthese, stabilized with $20 \mathrm{ppm}$ 4-tert-butylpyrocatechol), $40 \mathrm{~g}$ polyvinylpyrrolidone (PVP, Merck, $\mathrm{MW}: 25,000$ ) as steric stabilizer, $12 \mathrm{~g}$ sodium dodecylsulfate (SDS, Merck) as emulsifier, and $2.8 \mathrm{~g} \mathrm{4,4'-azo-bis(4-cyanopen-}$ tanoic acid) (ACPA, Fluka AG) as initiator. The polymerization was carried out in a $2 \mathrm{~L}$ vessel filled with $1,300 \mathrm{~mL}$ ethanol (chemical grade) at $70^{\circ} \mathrm{C}$. The mixture was stirred by a sixblade propeller stirrer at $200 \mathrm{rpm}$. After $8 \mathrm{~h}$ the reaction was terminated by lowering the temperature. This yielded approximately $210 \mathrm{~g}$ of PS particles. The particles were then washed six times in ethanol and twice in water by centrifugation. Finally they were redispersed in water and stored. Part of the PS was dried in a vacuum oven at $50^{\circ} \mathrm{C}$. Later on this part was used for dispersions in glycerol/water. As can be seen from Figure 1b, the particles were monodisperse. From electron micrographs it could be seen that all the particles were spherical. The numberaverage diameter were $1.61 \mu \mathrm{m}$ and the density $1,051 \mathrm{~kg} / \mathrm{m}^{3}$. The ratio of the weight average to the number-average diameter was 1.005 .

Dioctylphtalate p.a. (DOP) was obtained from Fluka AG and used as supplied. The density was $985 \mathrm{~kg} / \mathrm{m}^{3}$ and the viscosity at $25^{\circ} \mathrm{C}$ was $54.0 \mathrm{mPa} \cdot \mathrm{s}$. Glycerol (Merck, reinst) was used as supplied. It was mixed with double-distilled water to give a $86.1 / 13.9 \% \mathrm{w} / \mathrm{w}$ mixture with a density of $1.224 \mathrm{~kg} / \mathrm{m}^{3}$ and a viscosity of $140 \mathrm{mPa} \cdot \mathrm{s}$, both at $20^{\circ} \mathrm{C}$.

\section{Results and Discussion}

In Figures 2 to 5 relative viscosities as a function of shear rate are shown for the different dispersions investigated. All dispersions were stabilized by electrostatic stabilization. This can be concluded from $\zeta$-potential measurements (see below). It can be seen that both the dispersions of monodisperse and the dispersions of polydisperse particles show strong shear thickening.

The effect of polydispersity can be judged from comparison of results for polydisperse PVC particles with Hoffman's (1972) results for monodisperse PVC particles. Figure 6 shows that with polydisperse dispersions the increase in viscosity at the critical shear rate is still drastic but less dramatic and more gradual

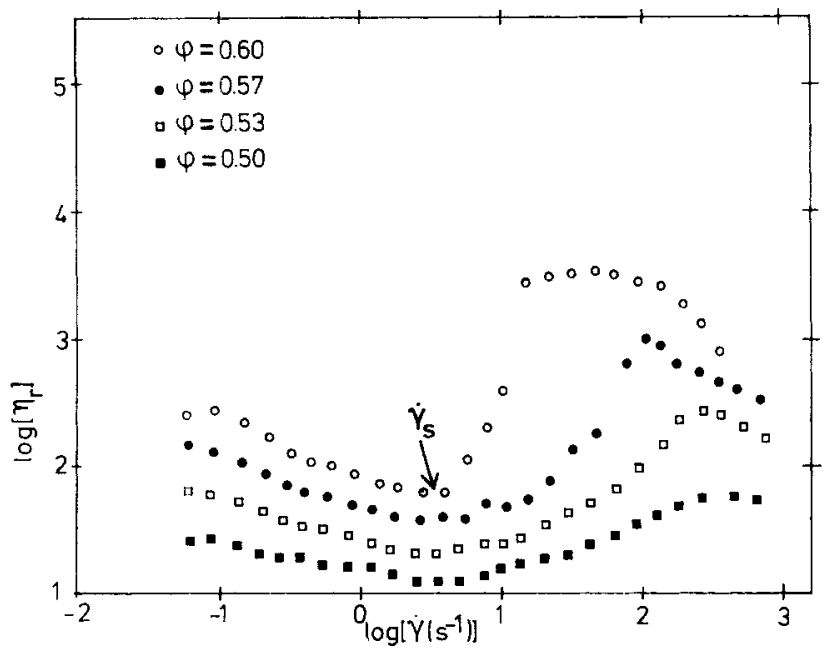

Figure 2a. Relative viscosities as a function of shear rate for dispersions of PVC in DOP at $25^{\circ} \mathrm{C}$.

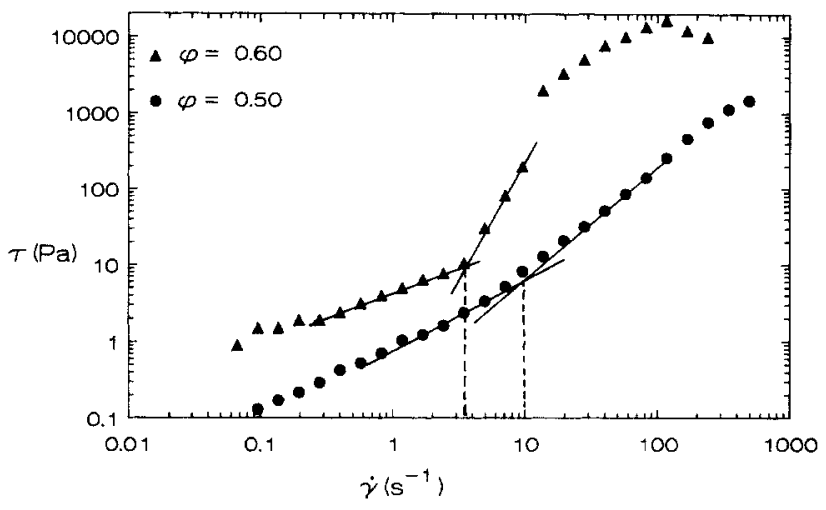

Figure 2b. Shear stress as a function of shear rate for two dispersions of PVC in DOP at $25^{\circ} \mathrm{C}$.

than with monodisperse dispersions. The measurements at low shear rates indicate a Newtonian region, suggesting absence of a yield stress with all dispersion systems except the PVC in DOP system, for which the Newtonian plateau is not clear. Care must be taken, however, with this conclusion because concentrated dispersions often show a quasi-Newtonian region that is preceded by a shear thinning region at lower shear rates. At high shear rates it was not possible to check the existence of a Newtonian plateau due to excessively high stress levels and because measurements are less accurate as a consequence of time-dependent effects. Therefore in this investigation we stopped measuring at values of the shear rate at which time-dependent effects started to appear. These effects might be due to shear-induced diffusion as reported by Leighton and Acrivos (1987), but more investigations are needed to clarify this point.

From the results presented it is possible to determine the shear rate at which shear thickening starts as judged from experiments (we define this shear rate as $\dot{\gamma}_{s, \text { meas }}$ ) for the different dispersions as a function of volume fraction. In Figure 2 the location of $\dot{\gamma}_{s, \text { meas }}$ is indicated at the curve for $\phi=0.60$. An accurate determination of the value of $\dot{\gamma}_{s}$ obviously is very difficult, especially at lower volume fractions. This problem can be solved partially by determining $\dot{\gamma}_{s}$ from a plot of the shear stress

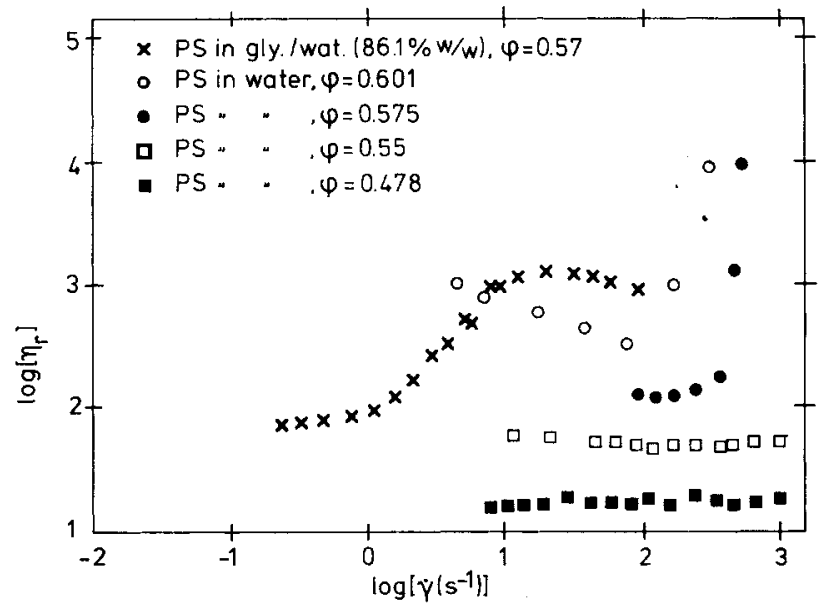

Figure 3. Relative viscosities as a function of shear rate for dispersions of PS in water and in glycerol/ water $(86.1 \% w / w)$ at $20^{\circ} \mathrm{C}$. 


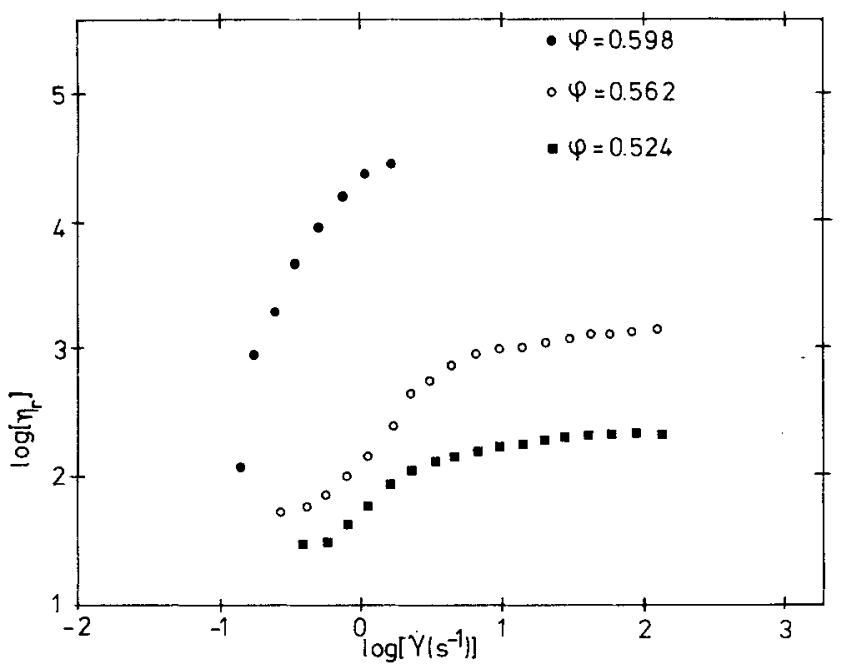

Figure 4. Relative viscosities for dispersions of $\mathrm{SiO}_{2}$ in glycerol/water $(86.1 \% \mathrm{w} / \mathrm{w})$ at $20^{\circ} \mathrm{C}$.

against the shear rate. This is shown in Figure $2 \mathrm{~b}$ for the highest and lowest volume fraction of our PVC dispersions. It can be seen that the curves for the shear stress can, in the neighborhood of $\dot{\gamma}_{s}$, be approximated by two straight lines. From the interception of these two lines the critical shear rate can be determined with much higher accuracy than from the viscosity plots. In Figure 7 values of $\dot{\gamma}_{s, \text { meas }}$ for all dispersions investigated are plotted against both volume fraction and dimensionless interparticle distance $h / a$ as calculated with Eq. 9. Values of $\dot{\gamma}_{s, \text { meas }}$ as deduced from the results of Hoffman (1972) are indicated as well.

The theoretical values of $\dot{\gamma}_{s}$ (denoted by $\dot{\gamma}_{s, c a l c}$ ) are calculated both at the appropriate volume fraction $\phi_{c}$ (simple model, Eqs. B2 and B3) and as functions of the volume fraction (refined model, Eqs. 5 and 7). For the systems investigated the parameters required in this calculation are shown in Table 1. Some data for our system of polydisperse PVC particles in DOP are not presented in the table because of lack of values for the $\zeta$-potential and the concentration of electrolyte in this system.

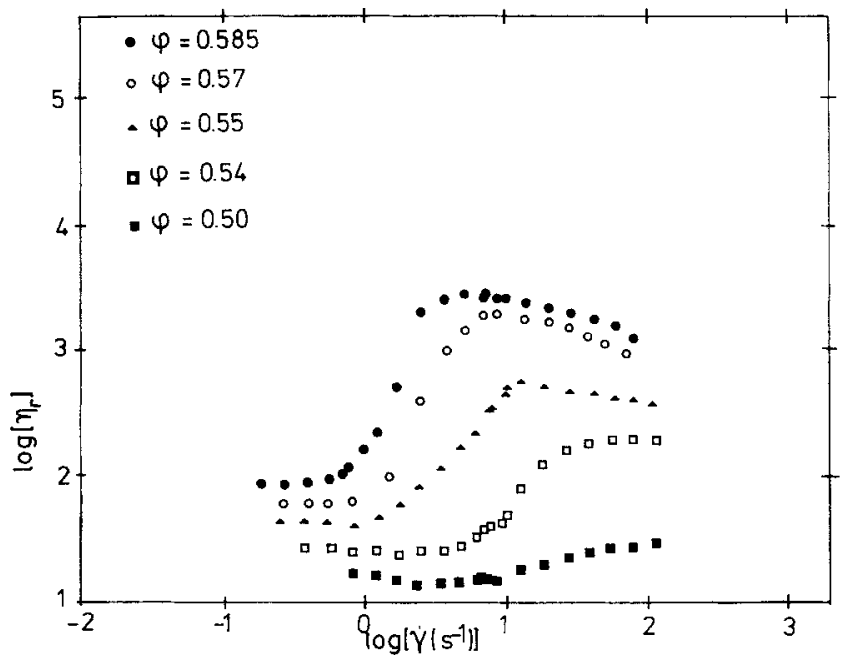

Figure 5. Relative viscosities for dispersions of glass in glycerol/water $(86.1 \% \mathrm{w} / \mathrm{w})$ at $20^{\circ} \mathrm{C}$.

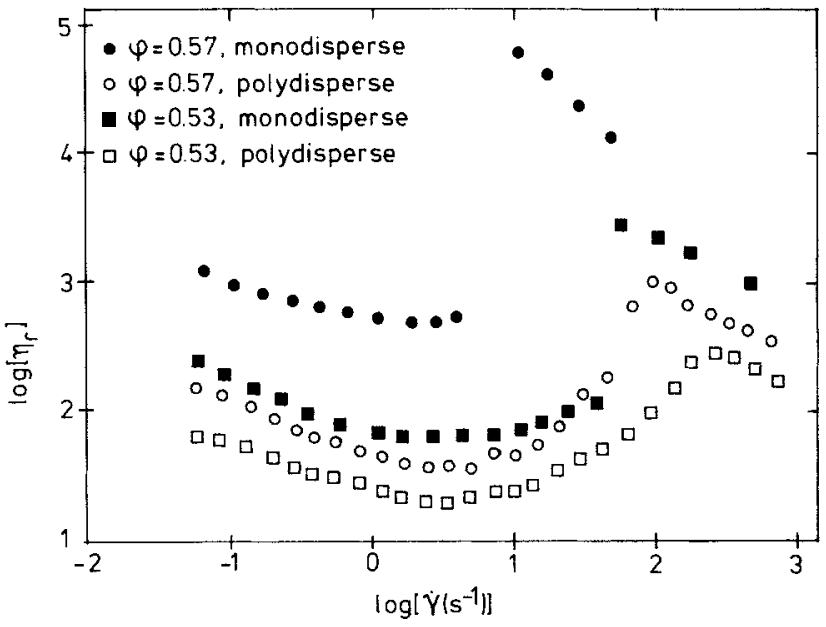

Figure 6. Relative viscosities for dispersions of polydisperse PVC in DOP compared to Hoffman's (1972) monodisperse results.

In Table 2 results from the simple model are compared with experimental results. Here too we give only the results for Hoffman's monodisperse PVC and not those for our polydisperse samples. As can be seen, the agreement between $\dot{\gamma}_{s, \text { meas }}$ and $\dot{\gamma}_{s, c a l c}$ is remarkably good. The values for $\dot{\gamma}_{s, \text { calc }}$ at $\phi_{c}$ are also shown in Figure 7 . These are the symbols surrounded by circles. The refined model indicates that $\dot{\gamma}_{s, \text { calc }}$ is proportional to the interparticle distance, Eq. 7. This theoretical dependence is expressed in the solid lines (with slope 1 in the double log plot) in Figure 7. The broken lines represent the experimentally determined dependences.

From the results for $\dot{\gamma}_{s, \text { meas }}$ at different volume fractions it can be seen that generally speaking the agreement is very good, both for the absolute magnitude of $\dot{\gamma}_{s}$ and for the slope of $\log \dot{\gamma}_{s}$ vs. $\log$ $h / a$. The agreement is less only for the slope in the case of polydisperse PVC dispersions. A possible explanation for this is that the polydisperse dispersion has a larger value of the maximum packing fraction $\phi_{\max }$ than 0.605 , as used in the calculations. A larger $\phi_{\max }$ in the calculations would increase this slope and improve the agreement with the theoretical dependence. In principle this correction should also be applied to the polydisperse glass dispersions, leading to a change in slope. However, this is not supported by the experimental resuits. This can be due to the presence of nonspherical particles, which in principle leads to a reduction of the maximum volume fraction. Experimentally it was found that the glass dispersions could not be prepared at volume fractions above approximately 0.6. The PVC dispersions, on the contrary, could be filled up to volume fractions above 0.6 .

To get another check of our model we performed some experiments at different temperatures on the dispersions of polystyrene particles in a glycerol/water mixture. By changing the temperature the viscosity of the medium could be varied and so the influence of $\eta_{0}$ on $\dot{\gamma}_{s}$ could be studied. Here we assume that in the limited temperature range studied, the influence of temperature on the parameters characterizing the electrostatic stabilization ( $\zeta$-potential, dielectric constant, and double layer thickness) is negligible compared to the influence of temperature on the viscosity of the dispersion medium. From thermal expansion coefficients (Perry and Chilton, 1973) it follows that the differ- 


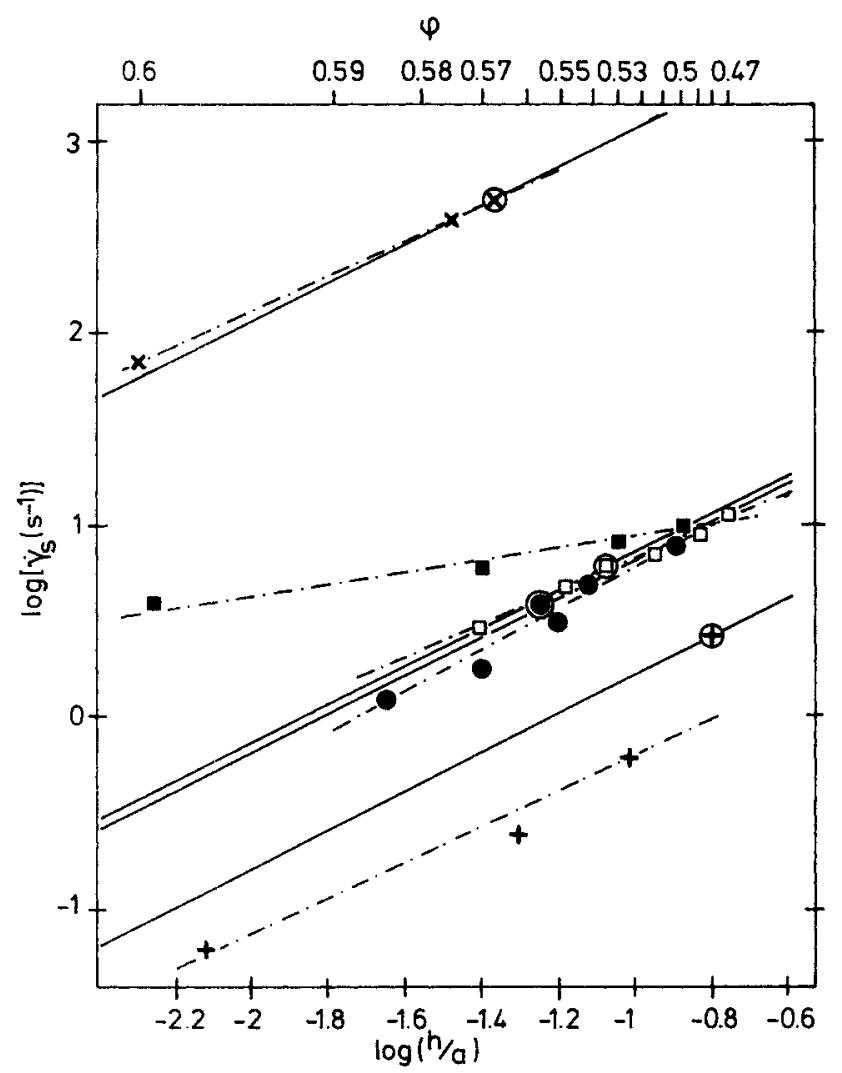

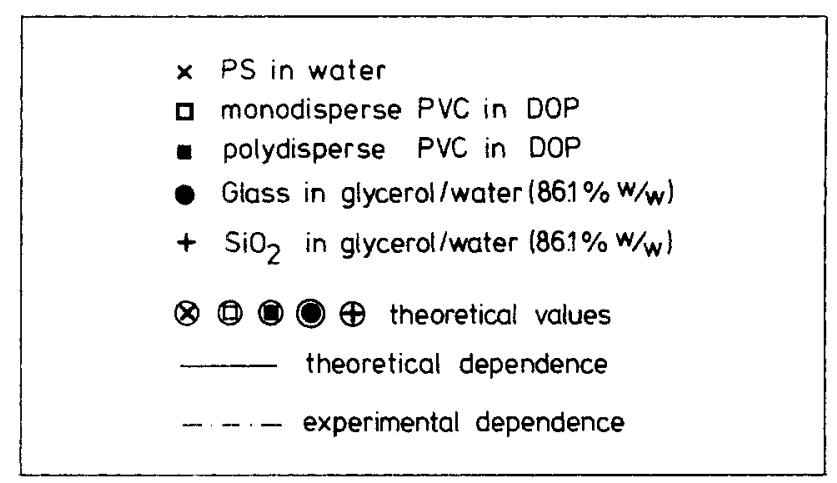

Figure 7. Critical shear rate as function of volume fraction and interparticle distance for various dispersions.

Experimental results (dashed lines, not encircled symbols) with theoretical models (encircled symbols: simple model; solid lines: refined model). ence in thermal expansion between polystyrene and glycerol/ water does not lead to significant changes in solid volume fraction. The results are given in Figure 8 . To make the graph more clear, for a few temperatures only smooth lines are shown. But also for these temperatures the spread of the measurements was comparable to the ones that are shown in Figure 8. The influence of temperature is mainly on $\dot{\gamma}_{s, m e a s}$ and, as far as the measurements indicate, hardly on $\eta_{r}(\dot{\gamma} \rightarrow 0)$ and on the maximum value of $\eta_{r}$. Horizontal shifting results in a fairly good covering of the curves. From these measurements values of $\log$ $\dot{\gamma}_{s, \text { meas }}$ can be obtained. In Figure 9 these values are plotted against $\log \eta_{0}$. From this figure it can be concluded that $\dot{\gamma}_{s, \text { meas }}$ is proportional to the inverse of $\eta_{0}$, in accordance with our theoretical model.

Some final remarks must be made about the uncertainty in the determination of several of the parameters. In this investigation the $\zeta$-potentials were determined in dilute dispersions. It is questionable to use these $\zeta$-potentials for the characterization of surface potentials in concentrated dispersions. For lack of better data, however, they can serve as a reasonable approximation. Another point is the determination of $x$. The ion content of the dispersions in glycerol/water was determined from a comparison of the conductivity of the supernatant with the conductivity of solutions of $\mathrm{KCl}$ in glycerol/water. The influence of the type of reference salt was checked by measuring conductivities of $\mathrm{BaCl}_{2}$ in glycerol/water as well. Equivalent amounts of $\mathrm{BaCl}_{2}$ and $\mathrm{KCl}$ (e.g., 0.05 and $0.1 \mathrm{~mol} / \mathrm{m}^{3}$, respectively) showed almost the same conductivities. This means that we do not make a large error when determining the value of $\kappa$ from the conductivities of $\mathrm{KCl}$ solutions.

Note that we did not take into account the self-screening effect as done, for example, by Russel and Benzing (1981). They performed an approximate, self-consistent field treatment of multiparticle interactions and showed that in ordered concentrated dispersions the value of $\kappa$ is underestimated when determining the ion content from the supernatant and using the dielectric constant of the pure fluid. Their theory is limited to situations in which the charge clouds around the particles are only very slightly disturbed by the small oscillatory shear imposed. This condition is fulfilled at frequencies much smaller than $\left(\epsilon_{0} \epsilon_{r} \eta_{0} k^{2} T^{2}\right) /\left(a^{2} e_{0}^{2} z^{2}\right)$. With all our dispersion systems the magnitude of this parameter is of the same order as the magnitude of the critical shear rate, which implies that the applicability of their theory is questionable. If one nevertheless uses their theory for obtaining a rough estimate of the influence of the suspension concentration effect on the extension of the electrical double layer in our systems, then one can calculate the value of

Table 1. Parameters for the Various Dispersions

\begin{tabular}{|c|c|c|c|c|c|c|c|}
\hline \multirow[b]{2}{*}{ Dispersion } & \multicolumn{7}{|c|}{ Parameter } \\
\hline & $\begin{array}{c}a \\
\mu \mathrm{m}\end{array}$ & $\begin{array}{c}\eta_{0} \\
\mathbf{P a} \cdot \mathbf{s}\end{array}$ & $\epsilon_{r}$ & $\begin{array}{l}T \\
\mathrm{~K}\end{array}$ & $\begin{array}{c}\psi_{0}(-\zeta) \\
m V\end{array}$ & $\underset{\mathrm{mol} \cdot \mathrm{m}^{-3}}{C}$ & $10^{7} \mathrm{~m}^{-}$ \\
\hline PVC in DOP, our sample & 0.7 & 0.054 & 5.2 & 298.2 & - & - & - \\
\hline PVC in DOP, Hoffman & 0.625 & 0.054 & 5.2 & 298.2 & 90 & 0.01 & 4.02 \\
\hline Glass in glycerin/water & 1.2 & 0.140 & 48.65 & 293.2 & 75 & 0.05 & 2.96 \\
\hline $\mathrm{SiO}_{2}$ in glycerin/water & 0.955 & 0.140 & 48.65 & 293.2 & 50 & 0.01 & 1.32 \\
\hline PS in glycerin/water & 0.805 & 0.140 & 48.65 & 293.2 & 31 & 0.2 & 5.92 \\
\hline PS in water & 0.805 & 0.001 & 80.37 & 293.2 & 37 & 0.3 & 5.64 \\
\hline
\end{tabular}

e, taken from Handbook of Chemistry and Physics. 
Table 2. Calculated and Measured Critical Shear Rate

\begin{tabular}{lccc}
\hline \multicolumn{1}{c}{ Dispersion } & $\phi_{c}$ & $\begin{array}{c}\dot{\gamma}_{s, \text { alc }} \\
\mathrm{s}^{-1}\end{array}$ & $\begin{array}{c}\dot{\gamma}_{\text {s,meas }} \\
\mathrm{s}^{-1}\end{array}$ \\
\hline PVC in DOP, Hoffman & 0.538 & 5.9 & 5 \\
Glass in glycerin/water & 0.557 & 4.0 & 3.2 \\
$\mathrm{SiO}_{2}$ in glycerin/water & 0.481 & 2.8 & 1.0 \\
PS in glycerin/water & 0.568 & 1.5 & 1.2 \\
PS in water & 0.567 & 504 & 480 \\
\hline
\end{tabular}

the critical parameter for deviations of $k$ from the equilibrium value. This parameter varies between $2 \times 10^{-4}$ and $3 \times 10^{-2}$, indicating that the Debye double layer thickness would not have been changed by double layer overlap. Thus we think that the use of the definition of $x$ in Eq. 2 is applicable to our concentrated dispersions.

\section{General Discussion}

The model that we derived provides us with a criterion for the onset of shear thickening that gives remarkably good comparison with experimental results and gives insight into the physical mechanisms responsible for shear thickening. For most dispersions the shear rate at which shear thickening starts (at a volume fraction dependent on the thickness of the stabilizing layer) was predicted very well. The error in $\dot{\gamma}_{s, c a l c}$ in most cases was not more than $25 \%$, which is acceptable in view of the uncertainties in the values of the $\zeta$-potential and the concentration of electrolyte. For almost all dispersions of spherical particles with stabilizing layer thickness smaller compared to the particle radius, at volume fractions close to the maximum packing fraction $\left(\phi_{\max }=0.605\right.$ for a freely flowing dispersion of monodisperse spherical particles with negligible Brownian motion) the dependence of $\dot{\gamma}_{s}$ on $\phi$ could also be predicted very well. It was shown that $\dot{\gamma}_{s}$ is proportional to the interparticle distance, which in this case is equal to the shortest distance between two layers of particles sliding over each other. This dependence follows from the balance between shear and interparticle forces when two particles are close together. The experimental results confirm this dependence.

For large double layers, irregularly shaped particles, and dispersions of polydisperse particles the method can be adapted by using another approach to the interparticle forces, adjusting the

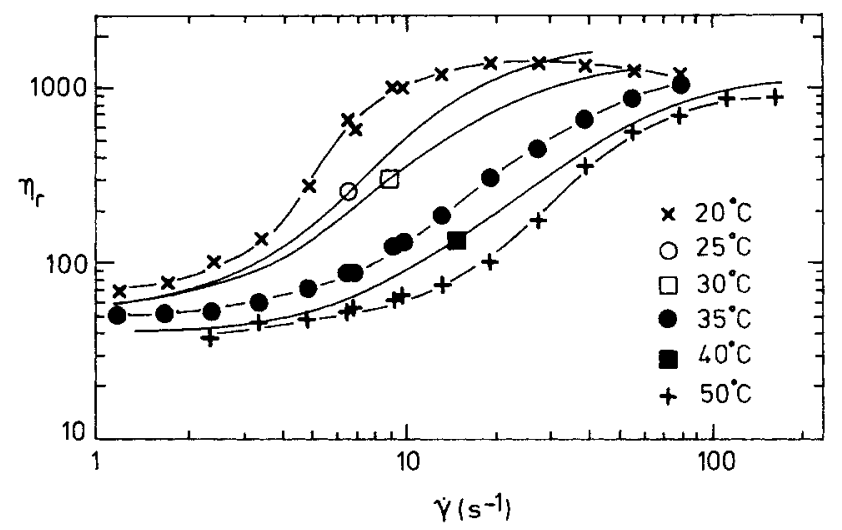

Figure 8. Relative viscosities as function of shear rate for dispersions of PS in glycerol/water $(86.1 \%$ w/ w), $\phi=0.57$, at various temperatures.

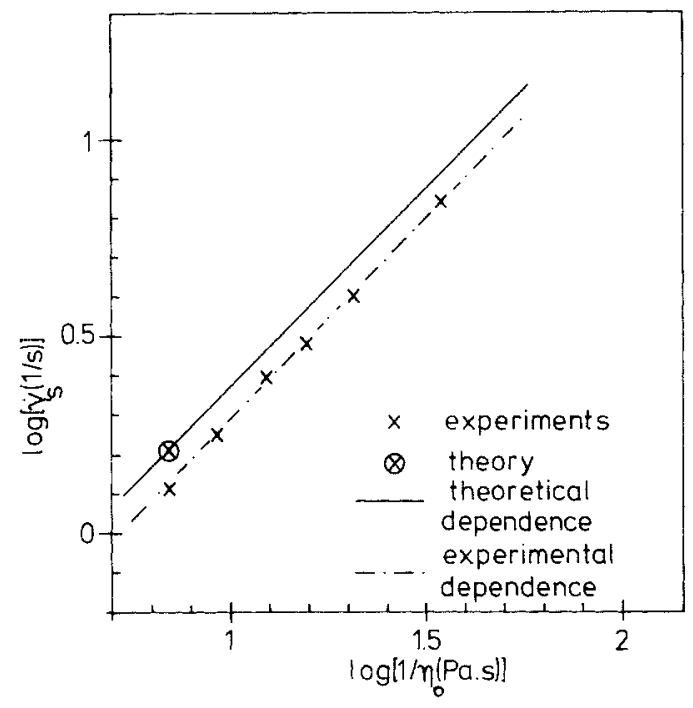

Figure 9. Dependence of critical shear rate on the inverse of dispersion medium viscosity.

modified Stokes law in Eq. 4, or by taking another maximum packing fraction for a free flowing dispersion.

Additional proof for the validity of the model also comes from the measurements of $\dot{\gamma}_{s}$ for the dispersion of PS in glycerol/ water at different temperatures and thus at different medium viscosities.

Laun (1988) measured the change of $\dot{\gamma}_{s}$ with change in $\mathrm{pH}$ (which implies change in $\zeta$-potential). He showed that the onset of shear thickening moved to lower shear rates when the $\mathrm{pH}$ was lowered (and thus the magnitude of the $\zeta$-potential decreased). Unfortunately there is not enough information to accurately calculate the $\dot{\gamma}_{s}$, but the trend is as would be expected; that is, the value of $\dot{\gamma}_{s}$ decreases when the $\zeta$-potential (and thus the stabilization) decreases. The same dependence of $\dot{\gamma}_{s}$ on $\zeta$-potential was observed by Umeya and Kanno (1979), who concluded that the shear thickening behavior results from the shear-induced association or flocculation of particles at a shear rate large enough to overcome the repulsive force between the particles in suspension. A similar conclusion was reached previously by Morgan (1968), who showed that shear thickening and colloidal stability are closely related.

Barnes (1989) concluded from all the data he collected on shear thickening dispersions that $\dot{\gamma}_{s}$ has inverse quadratic dependence on particle size. This would support our simple and our constant surface charge models. However, when replotting his graph of $\dot{\gamma}_{s}$ vs. $a$ in a graph of $\mu \dot{\gamma}_{s}$ vs. $a$ the quadratic dependence is much less clear. The data show, within experimental error, a linear relationship between $\mu \dot{\gamma}_{s}$ and $a$. This supports our constant surface potential model applied at a fixed volume fraction.

The observation that latices, without shear thickening, can slide in layers up to very high shear rates (Tomita and van de Ven, 1984) is not contradictory to our model. When the dimensionless number $N_{d}$ is calculated, Eq. 8 , for the dispersions of Tomita and van de Ven we get a maximum value of approximately 0.26 for $N_{d}$. We used their data of $a=249 \mathrm{~nm}, \psi_{0}=100$ $\mathrm{mV}, \dot{\gamma}=10^{4}, \eta_{0}=10^{-3}$, and $\epsilon_{r}=80.37$. However, for the transition of an ordered to a disordered state it is required that $N_{d}$ be larger than one. Additionally they used dispersions of relatively 
low volume fractions, below the volume fraction $\phi_{c}$ in Eq. B3, which will make the shear rate necessary for disorder even higher (our refined model).

Numerous other results in the literature seem to support the validity of our criterion for dilatancy. Unfortunately the authors almost never mentioned the parameters necessary to calculate $\dot{\gamma}_{s, c a l c}$. Nevertheless, with larger particles $\dot{\gamma}_{s, \text { meas }}$ moves to lower shear rates (Pätzold, 1980) and with sterically stabilized particles the dependence of $\dot{\gamma}_{s}$ on particle size is present as well (Wagstaff and Chaffey, 1977).

A last confirmation of our model is found in the results from computer simulations on sheared systems of particles that interact purely hydrodynamically (Bossis and Brady, 1984; Brady and Bossis, 1985). It is seen in these simulations that cluster formation takes place. No layer formation is detected. This agrees with our theory because Eq. 8 indicates that in this case $N_{d}$ becomes infinite. This means that ordered flow in layers will not occur, irrespective of the shear rate.

When comparing the results of our model with the force and torque balance presented by Hoffman (1974), a few striking differences appear. He predicted the dependence of $\dot{\gamma}_{s}$ on volume fraction (his Table I) to be much stronger than the dependence predicted by our model. Also the dependence of $\dot{\gamma}_{s}$ on the concentration of electrolyte, according to his model, is stronger than in our model (his Figure 5). The dependence of $\dot{\gamma}_{s}$ on the viscosity of the dispersion medium, however, is the same in both models. We tested how Hoffman's prediction of $\dot{\gamma}_{s}$ depends on the particle size and found (keeping all other parameters constant at representative levels) a maximum of $\dot{\gamma}_{s}$ at particle sizes of $\sim 0.2$ $1.0 \mu \mathrm{m}$. The fact that according to Hoffman $\dot{\gamma}_{s}$ decreases with decreasing particle size at the smaller size range is due to the van der Waals forces, which he took into account. However, at smaller particle sizes Brownian motion also is important. Because the energies of both effects scale identically with particle size, an improved model for smaller particles should analyze both effects. From the above discussion it can be concluded that, apart from the simpler scaling procedure in our model, there are also quite large quantitative differences between the two models.

We do not claim our model to be rigorous. However, the model gives a good indication of the parameters important in shear thickening and gives insight in to the processes occurring in a concentrated dispersion, especially with particles larger than $-0.5 \mu \mathrm{m}$. The right trends are predicted and the model can be useful to anyone who wants a quick estimate of the shear rate range in which a dispersion might show shear thickening. Ways to influence its occurrence in a practical situation may then be deduced from the model.

\section{Conclusions}

A model has been developed for the occurrence of shear thickening on the basis of a balance of hydrodynamic shear forces and stabilizing interparticle forces (either electrostatic or steric). The latter induces layer formation. In case the repulsive forces are of electrostatic nature, a dimensionless number, $N_{d}$, could be defined which characterizes the occurrence of shear thickening: shear thickening takes place if $N_{d}$ is comparable to or larger than one.

Experimental results on different dispersion systems confirm the validity of the model, especially the dependence of the criti- cal shear rate $\dot{\gamma}_{s}$ on the medium viscosity, the particle radius (literature results), and the volume fraction. Polydispersity hardly influences the critical value of $N_{d}$ but has a pronounced effect on the rise of the viscosity at $\dot{\gamma}_{s}$.

\section{Acknowledgment}

The authors wish to thank A. J. G, van Diemen for preparing the silica particles and P. J. M. Baets for measuring the dispersions of PS in water.

\section{Notation}

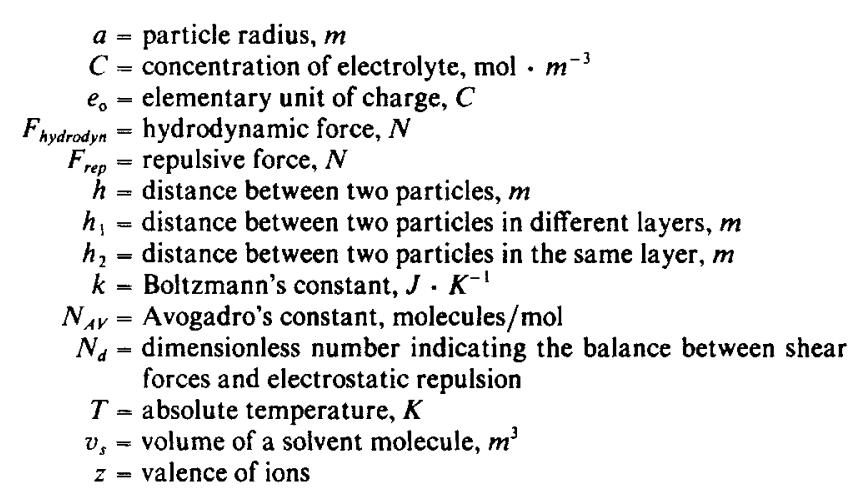

\section{Greek letters}

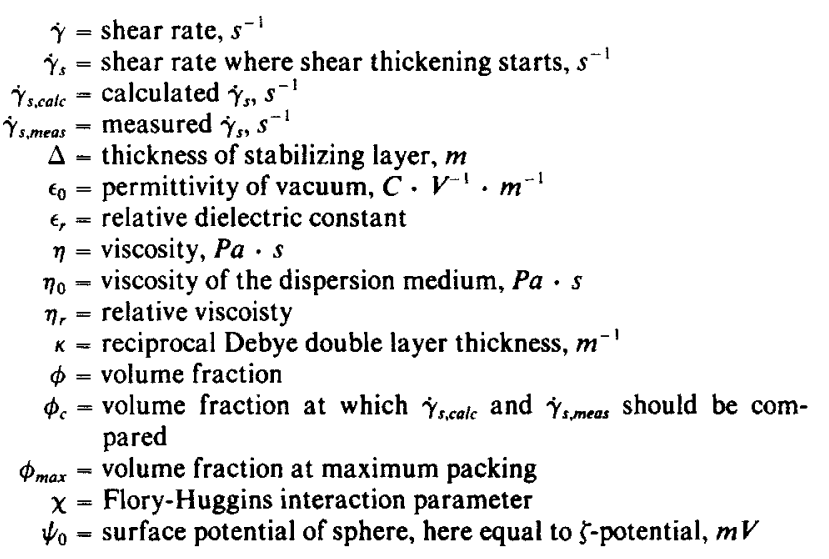

\section{Appendix A}

There are different methods of arranging particles in a layered dispersion and thus different ways of relating the volume fraction $\phi$ to a distance between particles $h$.

One method is to keep the distance between two layers equal to the characteristic distance between two particles in a layer. This characteristic distance $h$ is defined in the unit cell in Figure Al, with $h=h_{1}=h_{2}$. The volume of particles in the unit cell is $2 / 3 \pi a^{3}$. The volume of the unit cell is: $1 / 2 \cdot(2 a+h) \cdot 1 / 2 \sqrt{3}$. $(2 a+h) \cdot(2 a+h)$. This gives:

$$
\phi=\frac{2 / 3 \pi a^{3}}{1 / 4(2 a+h)^{3} \sqrt{3}}=\frac{8 \pi a^{3}}{3 \sqrt{3}(2 a+h)^{3}}
$$

or

$$
h=\left(\frac{8 \pi a^{3}}{3 \sqrt{3} \phi}\right)^{1 / 3}-2 a
$$




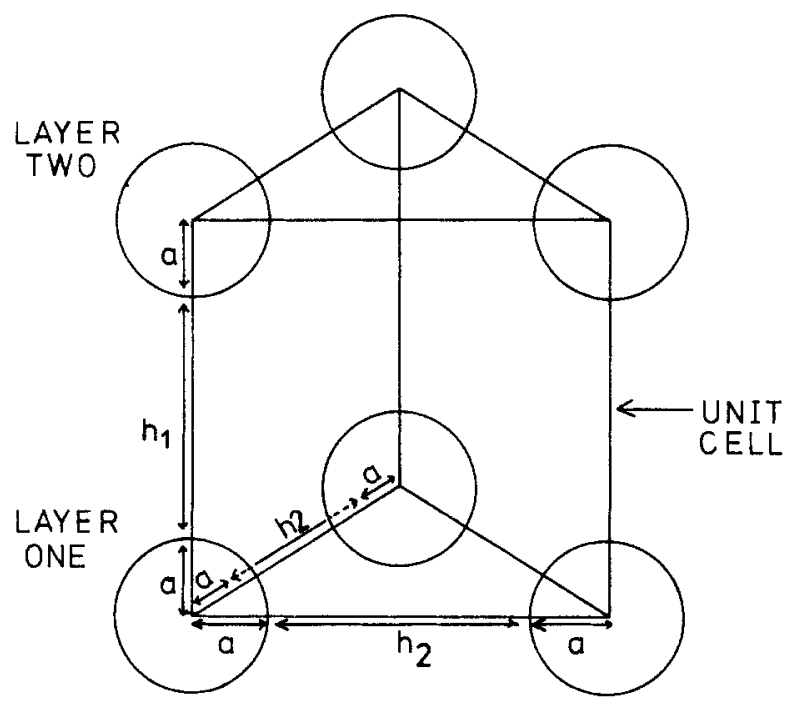

Figure A1. Unit cell for determination of the relation between volume fraction and distance between two particles.

A second method is to keep the particles closely packed in a layer while the distance between the layers is adjusted to $\phi$. We set $h=h_{1}$ and $h_{2}=0$ and get for the volume of the unit cell: $1 / 2$. $2 a \cdot 1 / 2 \sqrt{3} \cdot 2 a \cdot(2 a+h)$. This gives:

$$
\phi=\frac{2 / 3 \pi a^{3}}{\sqrt{3} a^{2}(2 a+h)}=\frac{2 \pi a}{3 \sqrt{3}(2 a+h)}
$$

or

$$
h=\frac{2 \pi a}{3 \sqrt{3} \phi}-2 a
$$

A third possibility is keeping the particles in a layer at shortest distance $2 / \kappa$ while adjusting the distance between layers. Then $h_{2}=2 / k$ and only the distance between the layers $\left(h_{1}=h\right)$ varies with varying volume fraction (as long as there is still space between two layers). For $\phi$ and $h$ this gives:

$$
\phi=\frac{8 \pi a^{3}}{3 \sqrt{3}(2 a+2 / \kappa)^{2}(2 a+h)}
$$

or

$$
h=\frac{8 \pi a^{3}}{3 \sqrt{3}(2 a+2 / k)^{2} \phi}-2 a
$$

The last method is less suitable because it can only be used for $h_{1}>h_{2}=2 / k$, thus at relatively low volume fractions. At higher volume fractions we must use one of the other approaches.

To decide which of these is the best approach the following facts must be noted. From computer simulations (Bossis and Brady, 1984) it can be seen that the distance between particles in a layer is approximately equal to the distance between layers. Additionally, Tomita and van de Ven (1984) showed experimentally that the layers of particles are not closely packed. Also they found that the distance between layers and thus the distance between particles in a layer varies with shear rate. On the other hand, Hoffman (1972) concluded from diffraction data that particles are closely packed within a layer. So there is uncertainty about the way particles are packed. Any of the three models mentioned above could be realistic. In view of these arguments we favor the first method, because it is the most flexible one. But it has to be mentioned that use of either of the other two approaches also signals the right trend and that the differences between the three approaches are quite small in comparison with the accuracy of the determination of $\kappa$.

\section{Appendix B}

We here present a simple model for the prediction of $\dot{\gamma}_{s}$, by considering simplified expressions for the magnitudes of the electrostatic and hydrodynamic forces. In doing this we neglect the dependence of $\dot{\gamma}_{s}$ on the volume fraction.

For electrostatically stabilized dispersions, equating the characteristic magnitudes of the interparticle and shear forces gives:

$$
6 \pi \eta_{0} a^{2} \dot{\gamma}=2 \pi \epsilon_{0} \epsilon_{r} \psi_{0}^{2}
$$

This gives as a first estimate for the critical shear rate $\dot{\gamma}_{s}$ :

$$
\dot{\gamma}_{s} \approx \frac{2 \pi \epsilon_{0} \epsilon_{r} \psi_{0}^{2}}{6 \pi \eta_{0} a^{2}}
$$

Another way of writing Eq. B2 is in the form of the dimensionless number in Eq. 8.

Actually, measured values of $\dot{\gamma}_{s}$ are dependent on the particle volume fraction $\phi$. Only if we know for which volume fraction Eq. B2 is the correct expression can we reasonably well compare the theoretical value of $\dot{\gamma}_{s}$ with measurements. To assess the volume fraction involved in the theoretical calculation we assume a specific regular packing of particles and then calculate the maximum volume fraction at which layers of particles still can slip along each other. A cubically stacked hexagonal packing seems a reasonable choice in view of the observation that its closest packing, where $\phi_{\max }$ equals approximately 0.605 , well matches experimental evidence of $\phi_{\max }$ for dispersions of monodisperse particles (Schreuder and Stein, 1988). In addition, light scattering by shear thickening dispersions suggests hexagonal packing (Hoffman, 1972; Tomita and van de Ven, 1984). Theoretically, pronounced shear thickening should also be observed in monodisperse dispersions with volume fractions above 0.605 . The ordered structure in these dispersions could then be a different one. However, these dispersions are not able to flow freely anymore and a continuous rearranging of the packing would have to take place. This was confirmed by our experiments. We were not able to make a well-flowing monodisperse dispersion with a volume fraction above 0.605 . In view of this we limit the applicability of our model to volume fractions for monodisperse dispersions below 0.605 .

If we now have a sphere with a diffuse double layer (electrostatic repulsion) the maximum repulsive forces manifest at an interparticle distance of the order of the double layer thickness, often captured in $1 / \kappa$. The value of $\kappa$ is given by $\mathrm{Eq} .2$.

We assume that the balance between hydrodynamic and stabilizing forces, Eq. B2, is not applicable to the volume fraction 
corresponding to touching spheres in a closest packing arrangement, but should be applied at a volume fraction with interparticle distances $2 / \kappa$. The new, corrected radius of the sphere then becomes $a+1 / \kappa$. The volume fraction that corresponds to a really free-flowing layered structure is:

$$
\phi_{c}=\left(\frac{a}{a+1 / \kappa}\right)^{3} \cdot 0.605
$$

This is the volume fraction at which we have to compare the measured and calculated $\dot{\gamma}_{s}$. Note that the choice for a fixed double layer thickness $1 / \kappa$ is an approximation, leading to simple relationships. In reality the distance at which the maximum repulsive force is found depends on $\psi_{0}, \kappa$, and the van der Waals forces.

Another way of arriving at Eq. B2 is by way of the method presented in the Theory section, but with an equation for the interaction force under constant surface charge conditions. For small $h \mathrm{Eq} .1$ then reduces to:

$$
F_{\text {rep }}=2 \pi \epsilon_{0} \epsilon_{r} \psi_{0}^{2} a / h
$$

Together with Eq. 4 this leads again to Eq. B2. According to this equation the critical shear rate does not depend on the volume fraction. The systems we investigated all showed dependence on volume fraction.

\section{Appendix $\mathbf{C}$}

For particles that are sterically stabilized the shear rate at which shear thickening starts can be calculated in a way analogous to that of electrostatically stabilized particles. The balance between stabilizing and shear forces can be written as (Russel, 1980; Osmond and Waite, 1975):

$$
\frac{4 / 3 \pi \Delta^{2}(1 / 2-\chi) k T}{v_{s}}=6 \pi \eta_{0} a^{2} \dot{\gamma}
$$

leading to

$$
\dot{\gamma}_{s} \approx \frac{4 / 3 \pi \Delta^{2}(1 / 2-\chi) k T}{6 \pi \eta_{0} a^{2} v_{s}}
$$

Here $\Delta$ is the thickness of the stabilizing layer, $\chi$ is the FloryHuggins interaction parameter, $v_{s}$ is the volume of a solvent molecule, and 0.605 is the maximum packing fraction for a freeflowing dispersion of spherical particles. The volume fraction at which Eq. C2 is applicable is given by:

$$
\phi_{c}=\left(\frac{a}{a+\Delta}\right)^{3} \cdot 0.605
$$

A problem with the formulas for steric stabilization is that measurement of the different variables involved is much more difficult than measurement of those involved in the case of electrostatic stabilization.

Another complication that may arise is the swelling of particles, leading to volume fractions not well defined but higher than anticipated. Additionally, some polymer chains from the particle can dissolve. This behavior was reported by Willey and Macosko (1982) for the system PVC in DOP. The solvated poly- mer chains raise the viscosity of the dispersion medium by about $20 \%$ and cause steric stabilization. However, Merinov et al. (1981) carefully measured the $\zeta$-potential of PVC in DOP and concluded that the electric double layer forces are responsible for the stabilization of the particles. Also, Lyklema (1968) reported that in media with low dielectric constants double layer forces can still be of considerable importance. Therefore we will use these forces in our calculations. So when more than one type of stabilization occurs in a system, the strongest one has to be taken for the calculations. Another example of this is the PS in water system we investigated. This is stabilized electrostatically as well as sterically. From the measured $\zeta$-potential and double layer thickness and the length of the stabilizing PVP chains, it can be estimated that the electrostatic stabilization is the strongest type of stabilization and so all the calculations must be done with this type of stabilization.

\section{Literature Cited}

Alince, B., and P. Lepoutre, "Flow Behavior of Pigment Blends," Tappi $J ., 66,57$ (1983).

Almog, Y., S. Reich, and M. Levy, "Monodisperse Polymeric Spheres in the Micron Size Range by a Single Step Process," Brit. Polym. J., 14, 131 (1982).

Barnes, H. A., "Shear Thickening (Dilatancy) in Suspensions of Nonaggregating Solid Particles Dispersed in Newtonian Liquids," J. Rheol., 33, 329 (1989).

Barnes, H. A., M. F. Edwards, and L. V. Woodcock, "Applications of Computer Simulations to Dense Suspension Rheology," Chem. Eng. Sci., 42, 591 (1987).

Berezov, L. V., F. D. Ovcharenko, and Yu. A. Merinov, "Factors Determining the Rheological Properties of Polyvinyl Chloride Plastisols," Koll. Zhu., 44, 10 (1982).

Bossis, G., and J. F. Brady, "Dynamic Simulation of Sheared Suspensions. I: General Method," J. Chem. Phys., 80, 5141 (1984).

Brady, J. F., and G. Bossis, "The Rheology of Concentrated Suspensions of Spheres in Simple Shear Flow by Numerical Simulation," J. Fluid Mech., 155, 105 (1985).

Brenner, H., "The Slow Motion of a Sphere Through a Viscous Fluid Toward a Plane Surface," Chem. Eng. Sci., 16, 242 (1961).

Denisov, I. E., A. I. Krasheninnikov, and V. G. Chervin, "Modeling Rheological Properties of a Suspension by the Molecular Dynamics Method," Koll. Zhu., 47, 790 (1985).

Durlofsky, L., J. F. Brady, and G. Bossis, "Dynamic Simulation of Hydrodynamically Interacting Particles," J. Fluid Mech., 180, 21 (1987).

Frankel, N. A., and A. Acrivos, "On the Viscosity of a Concentrated Suspension of Solid Spheres," Chem. Eng. Sci., 22, 847 (1967).

Frens, G., and J. Th. G. Overbeek, "Repeptization and the Theory of Electrocratic Colloids," J. Colloid Interf. Sci., 38, 376 (1972).

Heyes, D. M., "Shear Thinning and Thickening of the Lennard-Jones Liquid," J. Chem. Soc., Faraday Trans. 2, 82, 1365 (1986).

Hoffman, R. L., "Discontinuous and Dilatant Viscosity Behavior in Concentrated Suspensions. I: Observation of a Flow Instability," Trans. Soc. Rheol., 16, 155 (1972).

- "Discontinuous and Dilatant Viscosity Behavior in Concentrated Suspensions. II: Theory and Experimental Tests," J. Colloid Interf. Sci., 46, 491 (1974).

, "Discontinuous and Dilatant Viscosity Behavior in Concentrated Suspensions. III: Necessary Conditions for Their Occurrence in Viscometric Flows," Adv. Colloid Interf. Sci., 17, 161 (1982).

Kruyt, H. R., Colloid Science, Elsevier, Amsterdam (1952).

Laun, H. M., "Rheological Properties of Polymer Dispersions with Respect to Shear-induced Particle Structures," Progress and Trends in Rheology II, 287. Supplement to Rheologica Acta, H. Giesekus, ed., Proc. $2 d$ Conf. Eur. Rheologists, Prague, June 17-20, 1986 (1988).

Leighton, D., and A. Acrivos, "The Shear-induced Migration of Particles in Concentrated Suspensions," J. Fluid Mech., 181, 415 (1987).

Loose, W., and S. Hess, "Rheology of Dense Model Fluids via Nonequil- 
ibrium Molecular Dynamics: Shear Thinning and Ordering Transition," Rheol. Acta, 28, 91 (1989).

Lyklema, J., "Lyophobic Colloidal Dispersions," Adv. Colloid Interf. Sci, 2, 65 (1968).

Marrucci, G., and M. M. Denn, "On the Viscosity of a Concentrated Suspension of Solid Spheres," Rheol. Acta, 24, 317 (1985).

Merinov, Yu. A., V. V. Guzeev, L. V. Berezov, and M. N. Krupnova, "Electrokinetic Properties of Dispersions of a Pasteforming Polyvinylchloride in a Plasticizer," Koll. Zhu., 43, 487 (1981).

Metzner, A. B., and M. Whitlock, "Flow Behavior of Concentrated (Dilatant) Suspensions," Trans. Soc. Rheol., 2, 239 (1958).

Morgan, R. J., "A Study of the Phenomenon of Rheological Dilatancy in an Aqueous Pigment Suspension," Trans. Soc. Rheol., 12, 511 (1968).

Osmond, D. W. J., and F. A. Waite, "The Theoretical Basis for the Steric Stabilization of Polymer Dispersions Prepared in Organic Media," Dispersion Polymerization in Organic Media, K. E. J. Barrett, ed., Willey, London, Ch. 2, 9-44 (1975).

Pätzold, R., "Die Abhängigkeit des Fliessverhaltens Konzentrierter Kugelsuspensionen von der Strömungsform: Ein Vergleich der Viskosität in Scher- und Dehnströmungen," Rheol. Acta, 19, 322 (1980).

Perry, R. H., and C. H. Chilton, Chemical Engineers' Handbook, 5th ed., McGraw-Hill, New York (1973).

Russel, W. B., "Review of the Role of Colloidal Forces in the Rheology of Suspensions," J. Rheol., 24, 287 (1980).

Russel, W. B., and D. W. Benzing, "The Viscoelastic Properties of Ordered Latices: A Self-consistent Field Theory," J. Colloid Interf. Sci., 83, 163 (1981).

Schreuder, F. W. A. M., and H. N. Stein, 1988 "Rheology of Nonco- agulating Suspensions," Progress and Trends in Rheology II, 320. Supplement to Rheologica Acta, H. Giesekus, ed., Proc. $2 d$ Conf. Eur. Rheologists, Prague, June 17-20, 1986 (1988).

Strivens, T. A., "The Shear Thickening Effect in Concentrated Dispersion Systems," J. Colloid Interf. Sci., 57, 476 (1976).

Tan, C. G., B. D. Bowen, and N. Epstein, "Production of Monodisperse Colloidal Silica Spheres: Effect of Temperature," J. Colloid Interf. Sci., 118, 290 (1987).

Tomita, M., and T. G. M. Van De Ven, "The Structure of Sheared Ordered Latices," J. Colloid Interf. Sci., 99, 374 (1984).

Umeya, K., and T. Kanno, "Effect of Flocculation on the Dilatant Flow for Aqueous Suspensions of Titanium Dioxides," J. Rheol., 23, 123 (1979).

Verwey, E. G., and J. Th. G. Overbeek, Theory of the Stability of Lyophobic Colloids, Elsevier, Amsterdam (1948).

Wagstaff, I., and C. E. Chaffey, "Shear Thinning and Thickening Rheology. I: Concentrated Acrylic Dispersions," J. Colloid Interf. Sci., 59, 53 (1977).

Willey, S. J., and C. W. Macosko, "Steady Shear Rheological Behavior of PVC Plastisols," J. Rheol., 22, 525 (1978).

"Solvation Effects on Dilatancy in Concentrated PVC Plastisols," J. Rheol., 26, 557 (1982).

Woodcock, L. V., "Origins of Shear Dilatancy and Shear Thickening Phenomena," Chem. Phys. Lett., 111, 455 (1984).

Yoshimura, A., and R. K. Prud'homme, "Wall Slip Corrections for Couette and Parallel Disk Viscosimeters," J. Rheol., 32, 53 (1988).

Manuscript received July 17, 1989, and revision received Dec. 19.1989. 